\title{
GLOBAL SIZE OPTIMIZATION OF STATICALLY DETERMINATE TRUSSES CONSIDERING DISPLACEMENT, MEMBER, AND JOINT CONSTRAINTS
}

\author{
Roxane Van Mellaert ${ }^{1}$, Geert Lombaert, Mattias Schevenels
}

\begin{abstract}
Realistic truss design optimization problems are often governed by practical constraints. Because of the complexity of these constraints, usually only member constraints are taken into account during the optimization, and joint constraints are accounted for in a manual postprocessing step. This paper proposes a method to account for joint constraints in the global discrete size optimization of a steel truss structure. The design of an N-type truss girder is considered first without and then with the joint constraints specified in Eurocode 3. In order to guarantee global optimality in both cases, the optimization problem is reformulated as a mixed-integer linear program. A statically determinate analysis model is adopted so as to ensure that all joint constraints can be reformulated as linear functions. If the joint constraints are not considered in the optimization, a design is obtained where the joints need additional strengthening. This can be done by manually selecting heavier sections, which often leads to a suboptimal result, or by strengthening the joints (e.g. by means of stiffening plates), which has a serious impact on the fabrication cost. If the joint constraints are considered in the optimization, they are automatically satisfied by the final design. The weight of this design is about $15 \%$ higher than in the first case. This shows that the joint constraints have a significant impact on the optimal design. If the joint constraints are accounted for in a suboptimal way (e.g. by manually selecting heavier sections), the additional weight may be even higher. Taking into account joint constraints in the optimization leads to a cost reduction at two levels: in terms of engineering cost (no manual postprocessing step is needed) as well as fabrication cost (using unnecessarily heavy sections as well as joint strengthening are avoided).
\end{abstract}

Keywords: Truss design, discrete design optimization, joint constraints, mixed-integer linear program reformulation.

\section{INTRODUCTION}

Numerical optimization methods have a great potential to support structural engineers in finding the optimal design, and so to keep the consumption of natural resources of the building industry to a minimum. However, practicing structural engineers appear to be reluctant to adopt optimization as a daily design tool, even for relatively simple but tedious tasks such as the sizing of a steel truss girder. One of the reasons is that real-world design problems are often governed by a large number of constraints and practical issues. For a steel truss girder with welded joints, the usual displacement, member force, and buckling constraints as formulated in Eurocode 3 are imposed. In addition, the following practical constraints must be satisfied: the member sections must be chosen from a given section catalog, and the joints must obey certain geometrical rules in order to ensure structural integrity and weldability, as well as mechanical rules in order to avoid chord web failure, chord shear failure, and brace failure. Most existing design optimization algorithms cannot take into account all these practical constraints. As a consequence, a manual postprocessing step is required, where the optimized design is modified to satisfy the constraints which are not considered during the optimization. This operation is cumbersome, it costs precious engineering time, and it may lead to a suboptimal design or a design that no longer fulfills the stress and displacement constraints.

\footnotetext{
${ }^{1}$ Postprint submitted to Journal of Structural Engineering. Published version: R. Van Mellaert, G. Lombaert, M. Schevenels. Global Size Optimization of Statically Determinate Trusses Considering Displacement, Member, and Joint Constraints. J. Struct. Eng., 10.1061/(ASCE)ST.1943-541X.0001377, 04015120.
} 
A traditional approach to optimal truss design is to find a Fully Stressed Design (FSD): the element sections are iteratively updated until the stress in each element equals the maximum allowable value, which may lead to an optimal structural weight (Razani, 1965; Mueller et al., 2002). In its original formulation, the FSD method is only useful for stress constrained optimization problems. A modified Fully Utilized Design method (FUD) is proposed by Patnaik et al. (Patnaik et al., 1998) to take into account both stress and displacement constraints. Only in certain cases the result of the FSD method is optimal (Patnaik and Hopkins, 1998). However, for a practical steel truss design problem the joints remain to be designed manually. Dependent on the sections chosen for the braces and the chords, some of the joints will need to be strengthened by means of stiffening plates or by locally using a heavier section (Wardenier et al., 1992). Such interventions have little influence on the weight of the structure, but require additional welding as well as testing of the welds, and this has a significant impact on the fabrication cost.

An additional drawback of the FSD method is the fact that it only can handle continuous variables. For a practical steel truss design problem the profile of the members has to be chosen from a steel catalog, however. The optimization problem is therefore discrete. Several algorithms for discrete optimization have been proposed in the literature (Thanedar and Vanderplaats, 1995). The most popular algorithms that can handle discrete variables are evolutionary algorithms, such as simulated annealing (Balling, 1991), genetic algorithms (Camp et al., 1998; Rajeev and Krishnamoorthy, 1992), ant colony optimization (Camp et al., 2005), firefly algorithm (Gandomi et al., 2011), artificial bee colony algorithm (Sonmez, 2011), and particle swarm optimization (Venter and Sobieszczanski-Sobieski, 2003). These methods explore the design space in a random fashion, thereby using information collected from previous analyses to gradually move towards a better performing design. Evolutionary algorithms owe their popularity to the fact that they are easy to understand and to implement. They can cope with discrete parameters and are able to take into account complex constraints. However, evolutionary algorithms converge slowly, involve algorithmic parameters that require careful tuning, and global optimality cannot be guaranteed since no conclusive convergence checks can be made. In order to properly assess the influence of joint constraints on the optimal design of truss structures, it is important that global optimality can be guaranteed. Evolutionary algorithms are therefore not suitable.

The method used in this paper is to reformulate the optimization problem as a Mixed-Integer Linear Program (MILP), which is solved with the branch-and-bound method in order to achieve global optimality. This MILP is obtained by means of binary decision variables and the Simultaneous ANalysis and Design (SAND) approach: the state variables (the structural nodal displacements and the member end forces) are considered as additional design variables and the state equations (the equilibrium equations) are enforced by means of additional equality constraints. This optimization method has originally been proposed by Grossmann et al. (1992) for discrete size optimization problems and is extended by Rasmussen and Stolpe (2008) for truss topology design problems. Mela and Koski (2013) included all member constraints specified by the Eurocode in the truss topology design problem. In this paper, the focus is restricted to size optimization, but all relevant constraints precribed by the Eurocode (European Committee for Standardization, 2005a,b) are taken into account, including both the member constraints and the joint constraints.

In order to ensure that all joint resistance constraints can be reformulated as linear constraints in terms of the design variables, the scope of this paper is limited to statically determinate analysis models. Since the member forces of statically determinate models do not depend on the sections and remain constant in the optimization, they do not have to be considered as additional design variables. The joint constraints - some of which would be quadratic if the member forces are considered as design variables - can then be reformulated as mixed-integer linear constraints. Static determinacy implies that the structure is statically determined both internally and externally. Internal static 
determinacy is not a strong assumption as most trusses can be modeled with a sufficiently high accuracy assuming pinned connections. External static determinacy imposes a stronger limit on the applicability of the method as it only holds for simply supported trusses. It does not hold for continuous trusses or trusses that are part of a portal frame.

The example problem considered in this paper is the discrete size optimization of an N-type truss girder with welded joints under static nodal loading as shown in figure 1. The top chord members are steel H-sections (HEA), the bottom chord members are steel channel-sections (UPN), and the braces are steel Rectangular Hollow Sections (RHS). This N-type truss girder is widely used in practice.

The paper is organized as follows. In section 2, a summary of all governing design requirements for truss structures is given: the displacement, member force and buckling constraints as formulated in part 1-1 of Eurocode 3 (European Committee for Standardization, 2005a) as well as the joint resistance constraints as formulated in part 1-8 of Eurocode 3 (European Committee for Standardization, 2005b). In section 3, the mixed-integer linear formulation for statically determinate truss structures is introduced, and the example truss is optimized considering only displacement and member constraints. In section 4, the joint constraints are also taken into account. In section 5, the results are discussed. The optimal design without joint constraints and the optimal design with joint constraints are compared.

\section{DESIGN OF TRUSS STRUCTURES WITH WELDED JOINTS}

This section gives an overview of the design procedure for the example truss according to the European building codes.

First, a structural analysis is performed to obtain the member forces and the nodal displacements. Second, the imposed constraints are checked. In the serviceability limit state, the displacement constraints are verified. In the ultimate limit state, there are two types of capacity constraints: member constraints and joint constraints. The member constraints are specified in part 1-1 of Eurocode 3 and can be subdivided in member resistance constraints (to avoid yielding) and member stability constraints (to avoid buckling). The joint constraints are specified in part 1-8 of Eurocode 3 and can be subdivided in joint geometry constraints and joint resistance constraints. In addition, the global stability of the structure has to be checked, but in this paper it is assumed that the global stability is guaranteed by the secondary structure or by means of extra stiffeners.

\section{Structural models}

Two different models can be used for the structural analysis of trusses. In the first model, all members are pin connected as shown in figure 2a. As a consequence, only normal forces occur. This model provides a good approximation for trusses with slender members and where the centerlines of joined members intersect each other in a single point (Wardenier et al., 2008). Unfortunately, the latter is often not true for reasons of weldability and cutting. As a consequence, the intersection of the centerlines of the braces is located at a certain distance from the centerline of the chords. These nodal eccentricities cause bending moments in the chords, which are called eccentricity moments. The eccentricity moments have to be taken into account in the chord member design. As proposed in the CIDECT design guide (Wardenier et al., 2008), they are accounted for in an approximate way by distributing them equally over both chord members on either side of the joint.

In the second model, the chords are continuous and the braces are pin connected with infinitely stiff members at a distance of the eccentricity to the chords as shown in figure $2 \mathrm{~b}$. This model is internally statically indeterminate.

In order to ensure that all joint resistance constraints can be taken into account in the MILP formulation, only statically determinate models are considered. Therefore the first model is adopted in this paper. 


\section{Displacement constraints}

According to Eurocode 0, the vertical displacement $u_{z}$ of roof structures should be limited as follows:

$$
\left|u_{z}\right| \leqslant \frac{l}{200}
$$

where $l$ is the span of the structure.

\section{Member resistance constraints}

The capacities of the members are checked according to part 1-1 of Eurocode 3. The resistance of the cross-sections of the braces is checked as follows:

$$
\frac{|N| \gamma_{\mathrm{M} 0}}{A f_{\mathrm{y}}} \leqslant 1
$$

where $N$ is the design value of the normal force, $A$ is the cross-section area, $f_{\mathrm{y}}$ is the yield strength of the material, and $\gamma_{\mathrm{M} 0}$ is the partial factor for resistance of cross-sections, which is equal to 1.

The resistance of the cross-sections of the chords is checked as follows:

$$
\frac{|N| \gamma_{\mathrm{M} 0}}{A f_{\mathrm{y}}}+\frac{|M| \gamma_{\mathrm{M} 0}}{W_{\mathrm{pl}} f_{\mathrm{y}}} \leqslant 1
$$

where $M$ is the design value of the bending moment and $W_{\mathrm{pl}}$ is the plastic section modulus.

\section{Stability constraints}

The buckling resistance of members in compression and/or bending should be verified. Depending on the occurring forces - compression, bending, or both - and the section type, different types of buckling should be checked. For each buckling mode, a different reduction factor $\chi$ for the design resistance is calculated. All reduction factors are determined from the relevant buckling curve which is selected in agreement with the type of cross-section. The buckling curves depend on the section, the buckling length, and the yield strength of the material.

The flexural buckling resistance is checked as follows:

$$
\begin{aligned}
& -\frac{\chi_{y} A f_{\mathrm{y}}}{\gamma_{\mathrm{M} 1}} \leqslant N \\
& -\frac{\chi_{z} A f_{\mathrm{y}}}{\gamma_{\mathrm{M} 1}} \leqslant N
\end{aligned}
$$

where $\gamma_{\mathrm{M} 1}$ is the partial factor for resistance of members to instability, which is equal to 1 , and $\chi_{y}$ and $\chi_{z}$ are the reduction factors due to in-plane flexural buckling and out-of-plane flexural buckling.

For members in compression with open cross-sections such as HEA-sections, the torsional and the torsional-flexural buckling resistance have to be checked as well:

$$
\begin{array}{r}
-\frac{\chi_{\mathrm{T}} A f_{\mathrm{y}}}{\gamma_{\mathrm{M} 1}} \leqslant N \\
-\frac{\chi_{\mathrm{TF}} A f_{\mathrm{y}}}{\gamma_{\mathrm{M} 1}} \leqslant N
\end{array}
$$

where $\chi_{\mathrm{T}}$ and $\chi_{\mathrm{TF}}$ are the reduction factors due to torsional buckling and torsional-flexural buckling. 
For members bent in the plane of highest flexural rigidity the lateral-torsional buckling resistance should also be checked. For the HEA top chord members in bending and axial compression, the buckling resistance is checked as follows (assuming class 1 or class 2 cross-sections):

$$
\begin{aligned}
& \frac{|N| \gamma_{\mathrm{M} 1}}{\chi_{y} f_{\mathrm{y}} A}+k_{y y} \frac{|M| \gamma_{\mathrm{M} 1}}{\chi_{\mathrm{LT}} f_{\mathrm{y}} W_{\mathrm{pl}, y}} \leqslant 1 \\
& \frac{|N| \gamma_{\mathrm{M} 1}}{\chi_{z} f_{\mathrm{y}} A}+k_{z y} \frac{|M| \gamma_{\mathrm{M} 1}}{\chi_{\mathrm{LT}} f_{\mathrm{y}} W_{\mathrm{pl}, y}} \leqslant 1
\end{aligned}
$$

where $\chi_{\mathrm{LT}}$ is the reduction factor due to lateral-torsional buckling and $k_{y y}$ and $k_{z y}$ are interaction factors which can be determined according to one of the methods described in annex A or annex B of Eurocode 3. In this paper, the method described in annex B is used.

\section{Joint geometry constraints}

Each joint has to satisfy geometric constraints that either follow from practical considerations such as weldability or are imposed to ensure that the design remains within the range of validity of the joint capacity constraints discussed in the next subsection. When there is a gap between the braces that are connected to the chord, the joint is called a gap joint. When the braces overlap each other, the joint is called an overlap joint. To limit the eccentricity moments, the connections of the RHS braces and HEA top chords are chosen to be gap joints and the connections of the RHS braces and UPN bottom chords are chosen to be overlap joints. The two types of joints applied in the N-truss example as well as the notations used for all member dimensions are shown in figure 3. The notation used in the Eurocode is adopted: properties of chord members are denoted by a subscript 0 and properties of braces are denoted by the subscripts $i$ and $j$. For overlap joints, it is important to distinguish between the overlapping and the overlapped brace; here the subscript i refers to the overlapping brace and the subscript $\mathrm{j}$ to the overlapped brace. For the gap joints the gap $g$ has a positive value. In order to ensure weldability, the gap of the joint should be at least as large as the sum of the thicknesses $t_{\mathrm{i}}$ and $t_{\mathrm{j}}$ of the two braces. For the overlap joints the gap $g$ has a negative value. The overlap $\lambda_{\text {ov }}$ is chosen to be $100 \%$ for practical considerations: the end of the overlapping brace only has to be cut in one angle. As a consequence the overlap $g$ should be at least as large as the width $b_{\mathrm{i}}$ of the overlapping brace.

The geometry constraints specified in the Eurocode for the top joints read as: 


$$
\begin{aligned}
d_{\mathrm{w}} & \leqslant 400 \mathrm{~mm} \\
\frac{h_{\mathrm{i}}}{t_{\mathrm{i}}} & \leqslant 35 \\
\frac{h_{\mathrm{j}}}{t_{\mathrm{j}}} & \leqslant 35 \\
\frac{b_{\mathrm{i}}}{t_{\mathrm{i}}} & \leqslant 35 \\
\frac{b_{\mathrm{j}}}{t_{\mathrm{j}}} & \leqslant 35 \\
\frac{h_{\mathrm{i}}}{b_{\mathrm{i}}} & =1 \quad \text { (square section) } \\
\frac{h_{\mathrm{j}}}{b_{\mathrm{j}}} & =1 \quad \text { (square section) } \\
2.5 \mathrm{~mm} \leqslant t_{\mathrm{i}} & \leqslant 25 \mathrm{~mm} \\
2.5 \mathrm{~mm} \leqslant t_{\mathrm{j}} & \leqslant 25 \mathrm{~mm} \\
g & \geqslant t_{\mathrm{i}}+t_{\mathrm{j}}
\end{aligned}
$$

The geometry constraints for the bottom joints read as:

$$
\begin{aligned}
b_{0} & \leqslant 400 \mathrm{~mm} \\
\frac{h_{\mathrm{i}}}{t_{\mathrm{i}}} & \leqslant 35 \\
\frac{h_{\mathrm{j}}}{t_{\mathrm{j}}} & \leqslant 35 \\
\frac{b_{\mathrm{i}}}{t_{\mathrm{i}}} & \leqslant 35 \\
\frac{b_{\mathrm{j}}}{t_{\mathrm{j}}} & \leqslant 35 \\
0.5 \leqslant \frac{h_{\mathrm{i}}}{b_{\mathrm{i}}} & \leqslant 2 \\
0.5 \leqslant \frac{h_{\mathrm{j}}}{b_{\mathrm{j}}} & \leqslant 2 \\
2.5 \mathrm{~mm} \leqslant t_{\mathrm{i}} & \leqslant 25 \mathrm{~mm} \\
2.5 \mathrm{~mm} \leqslant t_{\mathrm{j}} & \leqslant 25 \mathrm{~mm} \\
\frac{b_{\mathrm{i}}}{b_{0}} & \geqslant 0.25 \\
\frac{b_{\mathrm{i}}}{b_{\mathrm{j}}} & \geqslant 0.75 \\
g & =-b_{\mathrm{i}}
\end{aligned}
$$

The constraint given by equation (31) imposes an overlap of $100 \%$ for practical considerations, which is stricter than the constraint specified in the Eurocode.

In addition, class 2 cross-sections must be used for the chords and class 1 cross-sections for the braces. 


\section{Joint resistance constraints}

For connections between hollow sections and open sections the following types of failure can occur: chord face failure, chord web failure, chord shear failure, punching shear failure, brace failure, and local buckling failure. According to part 1-8 of Eurocode 3, only certain failure modes must be considered for each type of joint. For HEA-RHS gap joints the following criteria should be checked: chord web failure, brace failure, and chord shear failure. For UPN-RHS overlap joints with an overlap of minimum $80 \%$ only brace failure of the overlapping brace member must be checked.

In order to avoid chord web failure in the HEA-RHS joints (figure 4), the normal force $N_{\mathrm{i}}$ in brace $\mathrm{i}$ is limited as follows:

$$
\left|N_{\mathrm{i}}\right| \leqslant \frac{f_{\mathrm{yc}} t_{\mathrm{w}} b_{\mathrm{w}}}{\sin \theta_{\mathrm{i}}} \frac{1}{\gamma_{\mathrm{M} 5}}
$$

where $f_{\mathrm{yc}}$ is the yield strength of the chord, $\gamma_{\mathrm{M} 5}$ is the partial safety factor for the resistance of joints in hollow section lattice girder, which is equal to 1 , and $b_{\mathrm{w}}$ is the effective width for the web of the chord, which is obtained as:

$$
b_{\mathrm{w}}=\min \left(\frac{h_{\mathrm{i}}}{\sin \theta_{\mathrm{i}}}+5\left(t_{\mathrm{f}}+r_{0}\right), 2 t_{\mathrm{i}}+10\left(t_{\mathrm{f}}+r_{0}\right)\right)
$$

The normal force $N_{\mathrm{j}}$ in brace $\mathrm{j}$ is limited in the same way.

In order to avoid brace failure in the HEA-RHS joints (figure 5), the normal force $N_{\mathrm{i}}$ in brace i is limited as follows:

$$
\left|N_{\mathrm{i}}\right| \leqslant 2 f_{\mathrm{yb}} t_{\mathrm{i}} p_{\mathrm{eff}} \frac{1}{\gamma_{\mathrm{M} 5}}
$$

where $f_{\mathrm{yb}}$ is the yield strength of the brace, and $p_{\mathrm{eff}}$ is the effective length of the contact area of the brace member onto the face of the chord, which is calculated as:

$$
p_{\mathrm{eff}}=\min \left(t_{\mathrm{w}}+2 r_{0}+7 t_{\mathrm{f}} \frac{f_{\mathrm{yc}}}{f_{\mathrm{yb}}}, b_{\mathrm{i}}+h_{\mathrm{i}}-2 t_{\mathrm{i}}\right)
$$

The normal force $N_{\mathrm{j}}$ in brace $\mathrm{j}$ is limited in the same way.

The last joint resistance check for the HEA-RHS joints is related to chord shear failure (figure 6). This failure mode is avoided by limiting the normal force $N_{\mathrm{i}}$ in brace $\mathrm{i}$ as follows:

$$
\left|N_{\mathrm{i}}\right| \leqslant \frac{f_{\mathrm{yc}} A_{\mathrm{v} 0}}{\sqrt{3} \sin \theta_{\mathrm{i}}} \frac{1}{\gamma_{\mathrm{M} 5}}
$$

where the shear area $A_{\mathrm{v} 0}=A_{0}-(2-\alpha) b_{0} t_{\mathrm{f}}+\left(t_{\mathrm{w}}+2 r\right) t_{\mathrm{f}}$, and $\alpha=\left(1+4 g^{2} / 3 t_{\mathrm{f}}^{2}\right)^{-\frac{1}{2}}$ for RHS sections. The normal force $N_{\mathrm{j}}$ in brace $\mathrm{j}$ is limited in the same way. In addition, the normal force of the chord $N_{0}$ is limited as follows:

$$
\left|N_{0}\right| \leqslant\left[\left(A_{0}-A_{\mathrm{v} 0}\right) f_{\mathrm{yc}}+A_{\mathrm{v} 0} f_{\mathrm{y} 0} \sqrt{1-\left(\frac{V}{V_{\mathrm{pl}, \mathrm{Rd}}}\right)^{2}}\right] \frac{1}{\gamma_{\mathrm{M} 5}}
$$

where the design shear force $V=\max \left(\left|N_{\mathrm{i}}\right| \sin \theta_{\mathrm{i}},\left|N_{\mathrm{j}}\right| \sin \theta_{\mathrm{j}}\right)$ and the plastic design shear resistance $V_{\mathrm{pl}, \mathrm{Rd}}=f_{\mathrm{y} 0} A_{\mathrm{v} 0} / \gamma_{\mathrm{M} 5} \sqrt{3}$.

For UPN-RHS overlap joints with an overlap of at least $80 \%$, only brace failure has to be checked (figure 7). This failure mode is avoided by limiting the normal force in the overlapping brace as follows: 


$$
\left|N_{\mathrm{i}}\right| \leqslant f_{\mathrm{yb}} t_{\mathrm{i}}\left(b_{\mathrm{i}}+b_{\mathrm{e}, \mathrm{ov}}+2 h_{\mathrm{i}}-4 t_{\mathrm{i}}\right) \frac{1}{\gamma_{\mathrm{M} 5}}
$$

where the effective width $b_{\mathrm{e}, \mathrm{ov}}$ of the connection between the overlapping brace and the overlapped brace is calculated as:

$$
b_{\mathrm{e}, \mathrm{ov}}=\min \left(\frac{10 t_{\mathrm{j}}^{2}}{b_{\mathrm{j}} t_{\mathrm{i}}} b_{\mathrm{i}}, b_{\mathrm{i}}\right)
$$

\section{OPTIMAL DESIGN WITHOUT JOINT CONSTRAINTS}

\section{Problem description}

In this section, the N-type truss girder described in the introduction and shown in figure 8 is optimized taking into account all member constraints. The objective of the optimization problem is to minimize the weight of the truss. The span is $l=20 \mathrm{~m}$, the height is $h=2 \mathrm{~m}$, the length of the chords and vertical braces is $L=2 \mathrm{~m}$, the angle of the vertical braces is $\theta_{\mathrm{i}}=90^{\circ}$, and the angle of the diagonal braces is $\theta_{\mathrm{j}}=45^{\circ}$. The value of the vertical load in the ultimate limit state is $F=100 \mathrm{kN}$ and is used to check the member and joint constraints, the value of the vertical load in the serviceability limit state is $F=74.07 \mathrm{kN}$ and is used to check the displacements. The maximum allowable displacement $u_{\max }$ is $l / 200=0.1 \mathrm{~m}$. All sections are steel sections with Young's modulus $E=210 \mathrm{GPa}$, and density $\rho=7850 \mathrm{~kg} / \mathrm{m}^{3}$.

The top chord sections are chosen from a catalog with twenty-four HEA-sections given in table 1 (ArcelorMittal, 2015). The bottom chord sections are chosen from a catalog with eighteen UPNsections given in table 2 (ArcelorMittal, 2015). The sections of the braces are chosen from a catalog with eighty-two cold formed square RHS-sections given in table 3 (van Eldik, 2006). All top chord members must have the same section, and all bottom chords members must have the same section. For the chords steel grade S355 is chosen and for the braces steel grade S275 is chosen. For the analysis of the truss, the model shown in figure 2a is adopted.

\section{Objective function and compatibility constraints}

In order to solve the discrete size optimization problem to global optimality, it is reformulated as a Mixed-Integer Linear Program (MILP). This approach was originally proposed to solve discrete size optimization problems (Grossmann et al., 1992) and was extended later for truss topology optimization problems (Stolpe and Svanberg, 2003; Stolpe, 2007; Rasmussen and Stolpe, 2008; Mela and Koski, 2013; Mela, 2013).

In order to obtain an MILP, the Simultaneous ANalysis and Design approach (SAND) is adopted. The design variables in the optimization problem are complemented with a set of continuous state variables, including the nodal displacements and normal forces, while the equilibrium equations are incorporated as equality constraints (Haftka, 1985; Arora and Wang, 2005), so no explicit structural analysis is made.

The analysis model adopted in this paper is statically determinate. The member forces are independent of the chosen sections and can be calculated a priori. As a consequence, the normal forces are not adopted as design variables and the equilibrium constraints are dropped. The original MILP proposed for discrete size optimization is thus simplified.

The design variables include a vector with binary decision variables $\mathbf{y}$ and a vector with continuous nodal displacement variables $\mathbf{u}$. The binary variables select a profile from the steel catalog. For each member $i$, section $j$ is selected from the set of available sections when the corresponding variable $y_{i j}=1$. Section $j$ is not selected for member $i$ when the corresponding variable $y_{i j}=0$. Although the number of optimization variables and constraints becomes large in this approach, the 
relaxed or continuous MILP is convex and can be efficiently solved to global optimality with existing solvers based on the branch-and-bound method.

In this subsection, the objective function and the compatibility constraints are formulated. In the following subsections, the Eurocode provisions regarding the maximum displacements and the strength of the members are added to the problem as inequality constraints in order to complete the MILP. In order to formulate the MILP for the optimization of the N-type truss, the members and catalogs of profiles are subdivided in different sets: the set of all members $\mathcal{M}$, all chord members $\mathcal{M}_{\mathrm{C}}$, top chord members $\mathcal{M}_{\mathrm{TC}}$, bottom chord members $\mathcal{M}_{\mathrm{BC}}$ and braces $\mathcal{M}_{\mathrm{B}}$, and the catalog of profiles $\mathcal{C}_{i}$ for member $i$. The optimization problem (without Eurocode constraints) is given by the following equations:

$$
\begin{aligned}
& \min _{\mathbf{y} \in \mathbb{B}^{n} \mathrm{~b}, \mathbf{u} \in \mathbb{R}^{n} \text { dof }} \rho \sum_{i \in \mathcal{M}} \sum_{j \in \mathcal{C}_{i}} L_{i} A_{i j} y_{i j} \\
& \text { s.t. } \\
& 1=\sum_{j \in \mathcal{C}_{i}} y_{i j} \quad \forall i \in \mathcal{M} \\
& \sum_{j \in \mathcal{C}_{i}} A_{i_{\mathrm{TC}}^{1} j} y_{i_{\mathrm{TC}}^{1} j}=\sum_{j \in \mathcal{C}_{i}} A_{i j} y_{i j} \quad \forall i \in \mathcal{M}_{\mathrm{TC}} \\
& \sum_{j \in \mathcal{C}_{i}} A_{i_{\mathrm{BC}}^{1} j} y_{i_{\mathrm{BC}}^{1} j}=\sum_{j \in \mathcal{C}_{i}} A_{i j} y_{i j} \quad \forall i \in \mathcal{M}_{\mathrm{BC}} \\
& \frac{E}{L_{i}} A_{i j} \mathbf{b}_{i}^{\mathrm{T}} \mathbf{u}-N_{i} \leqslant\left(1-y_{i j}\right) \bar{N}_{i j} \quad \forall i \in \mathcal{M}, \forall j \in \mathcal{C}_{i} \\
& \frac{E}{L_{i}} A_{i j} \mathbf{b}_{i}^{\mathrm{T}} \mathbf{u}-N_{i} \geqslant\left(1-y_{i j}\right) \underline{N}_{i j} \quad \forall i \in \mathcal{M}, \forall j \in \mathcal{C}_{i}
\end{aligned}
$$

The objective is to minimize the weight of the truss structure as expressed by equation (39). In this equation $\rho$ is the density of the material which is assumed to be the same for all members, $L_{i}$ is the length of member $i$, and $A_{i j}$ is the section area of section $j$ for member $i$. The constraint in equation (40) ensures that a single section $j$ is chosen from the catalog $\mathcal{C}_{i}$ for member $i$. Equations (41) and (42) ensure that all top chord members and all bottom chord members have the same section, where $i_{\mathrm{TC}}^{1}$ refers to the first top chord member and $i_{\mathrm{BC}}^{1}$ refers to the first bottom chord member. Equations (43) and (44) are compatibility constraints used to calculate the displacements $\mathbf{u}$. In these equations, $E$ is the Young's modulus of the material, $\mathbf{b}_{i}$ is the statics vector of member $i$ (Rasmussen and Stolpe, 2008) that accounts for the location and orientation of the members, $N_{i}$ is the normal force in member $i$, and $\bar{N}_{i j}$ and $\underline{N}_{i j}$ are artificial upper and lower bounds introduced to ensure feasibility of the optimization problem when section $j$ is not selected for element $i$ and $y_{i j}=0$. In this example, the values of the upper and lower bounds of the compatibility constraints in equations (43) and (44) are calculated based on the minimum and maximum allowed displacement (Rasmussen and Stolpe, 2008).

The total number of members in the structure is denoted by $n_{\mathrm{m}}$, the total number of available sections for each member $i$ is denoted by $n_{\mathrm{s}, i}$, the total number of degrees of freedom is denoted by $n_{\mathrm{dof}}$, and the total number of joints is denoted by $n_{\mathrm{g}}$. As a consequence, the total number of binary decision variables is $n_{\mathrm{b}}=\sum_{i=1}^{n_{\mathrm{m}}} n_{\mathrm{s}, i}$.

\section{Displacement constraints}

The displacement constraints are given by equation (45). Here $\underline{\mathbf{u}}=-0.1 \mathrm{~m}$ and $\overline{\mathbf{u}}=0.1 \mathrm{~m}$ are the minimum and maximum allowed displacement, respectively. 


$$
\underline{\mathbf{u}} \leqslant \mathbf{u} \leqslant \overline{\mathbf{u}}
$$

\section{Member constraints}

In this subsection, the member resistance and stability constraints described in the previous section are incorporated in the MILP. The joint constraints are not considered yet; it is therefore assumed that the members can be connected in such a way that eccentricities are avoided. No eccentricity moments are therefore taken into account in the optimization.

The stress constraints given by equation (2) and the in-plane and out-of-plane flexural buckling, torsional buckling and torsional-flexural buckling constraints given by equations (4) to (7) are reformulated as:

$$
\begin{aligned}
& N_{i} y_{i j} \leqslant f_{\mathrm{yc}} A_{i j} y_{i j} \quad \forall i \in \mathcal{M}_{\mathrm{C}}, \forall j \in \mathcal{C}_{i} \\
& N_{i} y_{i j} \geqslant-\min \left(1, \chi_{y}, \chi_{z}, \chi_{\mathrm{T}}, \chi_{\mathrm{TF}}\right) f_{\mathrm{yc}} A_{i j} y_{i j} \quad \forall i \in \mathcal{M}_{\mathrm{C}}, \forall j \in \mathcal{C}_{i} \\
& N_{i} y_{i j} \leqslant f_{\mathrm{yb}} A_{i j} y_{i j} \quad \forall i \in \mathcal{M}_{\mathrm{B}}, \forall j \in \mathcal{C}_{i} \\
& N_{i} y_{i j} \geqslant-\min \left(1, \chi_{y}, \chi_{z}\right) f_{\mathrm{yb}} A_{i j} y_{i j} \quad \forall i \in \mathcal{M}_{\mathrm{B}}, \forall j \in \mathcal{C}_{i}
\end{aligned}
$$

where $f_{\mathrm{yc}}$ and $f_{\mathrm{yb}}$ are the yield strength of the chords and braces, respectively. Following the recommendations in the CIDECT guide (Wardenier et al., 1992), the buckling lengths are assumed as follows: for the chords $L_{\mathrm{cr}, \mathrm{y}}=L_{\mathrm{cr}, \mathrm{z}}=0.9 L_{i}$, and for the braces $L_{\mathrm{cr}, \mathrm{y}}=L_{\mathrm{cr}, \mathrm{z}}=0.75 L_{i}$. The constraints in equations (46) to (49) are linear equations in terms of the binary decision variables $y_{i j}$.

\section{Results}

The MILP given by equations (39) to (49) consists of 1133 design variables, including 1112 binary design variables and 21 continuous design variables, 4519 constraints, and 11698 non-zeros. The MILP is solved by means of Gurobi 5.6 (Gurobi Optimization Inc., 2013), which uses the cutand-branch method, on a computer with a 4 threads Intel Core(TM)2 Quad CPU Q9550 processor and $4.0 \mathrm{~GB}$ RAM. The results are given in table 4 . The total weight of the N-truss is $1826.3 \mathrm{~kg}$. The nodal displacements are given in table 5. It is observed that the displacement constraints are not critical. Gurobi is capable of solving this problem in the preprocessing stage. Without performing a presolve or generating cuts, the problem is solved also at the root node. The computation time is less than 0.2 seconds in both cases. This is probably due to the fact that the displacement constraints do not become active. Without displacement constraints, the problem becomes trivial due to the static determinacy of the truss. Therefore, it is possible to verify the solution: the minimum section areas that are required according to the member strength and stability constraints can be calculated manually. This leads to the same results.

It is now verified to what extent the optimized design satisfies the constraints that are not explicitly considered in the MILP. In order to ensure weldability of the joints, minimal eccentricities are introduced as specified in table 6 . The gaps of the top joints are chosen to be equal to the sum of the thickness of the two braces of the joint. The gaps of the bottom joints are chosen so that the overlap is $100 \%$ in order to avoid difficult cutting of the overlapping brace. Table 7 gives the utilization ratios for all the constraints that must be checked according to part 1-1 and part 1-8 of Eurocode 3 as described in previous section. These utilization ratios are calculated as the ratio between the actual value and the maximum allowed value. Due to the introduction of nodal eccentricities after the optimization, the stability ratios are not guaranteed to be smaller than 1 . However, as can be observed in table 7 , the effect of the eccentricities is limited and all stability constraints remain satisfied. On the other hand, several utilization ratios of the joint constraints 
exceed the maximum allowed value of 1 , which are indicated in bold in table 7 . As a consequence, the joints have to be strengthened in order to satisfy all constraints. This can be done by either selecting different profiles, or by locally strengthening the joints e.g. by means of stiffening plates. The first approach would lead to a suboptimal result, as it is very difficult to determine which members should be made heavier and which sections should be selected. The second approach would only have a limited impact on the weight of the truss, but the fabrication costs would be much higher.

\section{OPTIMAL DESIGN WITH JOINT CONSTRAINTS}

\section{Problem description}

In this section, the displacement constraints, the member constraints, and the joint constraints are considered in the optimization. Due to the introduction of the joint geometry constraints, trusses with zero eccentricities become infeasible - non-zero eccentricities are therefore allowed. In order to ensure that the impact of the eccentricities on the weight of the truss remains minimal, the gap sizes (which control the eccentricities) are considered as additional design variables in the optimization problem. As a consequence, the design variables now consist of the nodal displacements $\mathbf{u} \in \mathbb{R}^{n}$ dof, the binary decision variables $\mathbf{y} \in \mathbb{B}^{n_{\mathrm{b}}}$, and the gaps of the joints $\mathbf{g} \in \mathbb{R}^{n_{\mathrm{g}}}$, where $n_{\mathrm{g}}$ is the number of joints.

\section{Objective function and compatibility constraints}

In this subsection, the objective function and the compatibility constraints are formulated. In the following subsections, the Eurocode provisions regarding the maximum displacements, the strength of the members, and the strength of the joints are added to the problem as inequality constraints in order to complete the MILP. In order to formulate the MILP for the optimization of the N-type truss, the members and catalogs of profiles are subdivided in different sets: the set of all members $\mathcal{M}$, all chord members $\mathcal{M}_{\mathrm{C}}$, top chord members $\mathcal{M}_{\mathrm{TC}}$, bottom chord members $\mathcal{M}_{\mathrm{BC}}$ and braces $\mathcal{M}_{\mathrm{B}}$, and the catalog of profiles $\mathcal{C}_{i}$ for member $i$. The joints are subdivided into different sets: the set of all joints is denoted by $\mathcal{J}$, the set of all joints with a chord on the left-hand side is denoted by $\mathcal{J}_{\mathrm{CL}}$, the set of all joints with a chord on the right-hand side is denoted by $\mathcal{J}_{\mathrm{CR}}$, the set of all top joints is denoted by $\mathcal{J}_{\mathrm{TJ}}$, and the set of all bottom joints is denoted by $\mathcal{J}_{\mathrm{BJ}}$. The optimization problem (without Eurocode constraints) is given by the following equations:

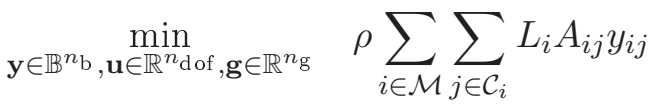

$$
\begin{aligned}
& \text { s.t. } \quad 1=\sum_{j \in \mathcal{C}_{i}} y_{i j} \quad \forall i \in \mathcal{M} \\
& \sum_{j \in \mathcal{C}_{i}} A_{i_{\mathrm{TC}}^{1}} y_{i_{\mathrm{TC}}^{1}}=\sum_{j \in \mathcal{C}_{i}} A_{i j} y_{i j} \quad \forall i \in \mathcal{M}_{\mathrm{TC}} \\
& \sum_{j \in \mathcal{C}_{i}} A_{i_{\mathrm{BC}}^{1}} y_{i_{\mathrm{BC}}^{1}}=\sum_{j \in \mathcal{C}_{i}} A_{i j} y_{i j} \quad \forall i \in \mathcal{M}_{\mathrm{BC}} \\
& \frac{E}{L_{i}} A_{i j} \mathbf{b}_{i}^{\mathrm{T}} \mathbf{u}-N_{i} \leqslant\left(1-y_{i j}\right) \bar{N}_{i j} \quad \forall i \in \mathcal{M}, \forall j \in \mathcal{C}_{i} \\
& \frac{E}{L_{i}} A_{i j} \mathbf{b}_{i}^{\mathrm{T}} \mathbf{u}-N_{i} \geqslant\left(1-y_{i j}\right) \underline{N}_{i j} \quad \forall i \in \mathcal{M}, \forall j \in \mathcal{C}_{i}
\end{aligned}
$$

These equations are identical to equations (39) to (44) for the case without joint constraints, except that the gaps are now also considered as design variables. 


\section{Displacement constraints}

The displacement constraints are identical to the displacement constraints given by equation (45) in previous section:

$$
\underline{\mathbf{u}} \leqslant \mathbf{u} \leqslant \overline{\mathbf{u}}
$$

\section{Member constraints}

Since the eccentricity moments depend on the gap sizes, the mixed-integer linear reformulation of the combined stress and stability constraints depends not only on the binary decision variables $y_{i j}$, but also on the gap variables $g_{m}$. The resistance of the cross-sections of the chords is checked according to equation (3). The design values of the normal forces are obtained before optimization. The design values of the bending moments, however, depend on the eccentricities, which depend on the gap size $g_{m}$. The eccentricity $e_{m}$ in joint $m$ is calculated as (Wardenier et al., 1992):

$$
e_{m}=\frac{g_{m}+\frac{b_{i_{m}^{\mathrm{BL}}}}{2 \sin \theta_{i_{\mathrm{BL}}}}+\frac{b_{i_{m}^{\mathrm{BR}}}}{2 \sin \theta_{i_{m}^{\mathrm{BR}}}}}{\tan \theta_{m}}-\frac{h_{i_{m}^{\mathrm{CR}}}}{2}
$$

where $i_{m}^{\mathrm{BL}}$ is the index of the left or overlapped brace in joint $m, i_{m}^{\mathrm{BR}}$ is the index of the right or overlapping brace in joint $m, i_{m}^{\mathrm{CR}}$ is the index of the right chord in joint $m$, and $\theta_{m}$ is the angle between the two braces of the joint, which is calculated as $\theta_{m}=\pi-\theta_{i_{m}^{\mathrm{BL}}}-\theta_{i_{m}^{\mathrm{BR}}}$. The eccentricity moment $M_{\mathrm{e}, m}$ in joint $m$ is calculated as (Wardenier et al., 1992):

$$
M_{\mathrm{e}, m}=\frac{\Delta N_{m} e_{m}}{2}
$$

where $\Delta N_{m}=\left|N_{i_{m}^{\mathrm{CL}}}-N_{i_{m}^{\mathrm{CR}}}\right|$ denotes the change in normal force in the chord at joint $m, i_{m}^{\mathrm{CL}}$ is the index of the left chord in joint $m$, and $i_{m}^{\mathrm{CR}}$ is the index of the right chord in joint $m$.

For simplicity, the linear forms of the member constraints are first derived for the case where we have only a single available profile for each member. In this case, the resistance of the cross-sections of the chords at the right-hand side of the joint is checked according to equation (3) as follows:

$$
\frac{\left|N_{i_{m}^{\mathrm{CR}}}\right|}{f_{\mathrm{yc}} A_{i_{m}^{\mathrm{CR}}}}+\frac{\left|M_{\mathrm{e}, m}\right|}{f_{\mathrm{yc}} W_{\mathrm{pl}, y, i_{m}^{\mathrm{CR}}}} \leqslant 1
$$

where $f_{\mathrm{yc}}$ is the yield strength of the chords, $W_{\mathrm{pl}, y, i_{m}^{\mathrm{CR}}}$ is the plastic section modulus along the y-axis, and the safety factors are dropped as they are equal to 1. Replacing the bending moment $\left|M_{\mathrm{e}, m}\right|$ by the expression given by equation (58) gives:

$$
\frac{\left|N_{i_{m}^{\mathrm{CR}}}\right|}{f_{\mathrm{yc}} A_{i_{m}^{\mathrm{CR}}}}+\frac{\Delta N_{m}\left|e_{m}\right|}{2 f_{\mathrm{yc}} W_{\mathrm{pl}, y, i_{m}^{\mathrm{CR}}}} \leqslant 1
$$

Replacing the eccentricity $\left|e_{m}\right|$ by the expression given by equation (57) gives:

$$
\frac{\left|N_{i_{m}^{\mathrm{CR}}}\right|}{f_{\mathrm{yc}} A_{i_{m}^{\mathrm{CR}}}}+\frac{\Delta N_{m}}{2 f_{\mathrm{yc}} W_{\mathrm{pl}, y, i_{m}^{\mathrm{CR}}}}\left|\frac{g_{m}+\frac{b_{i_{m}^{\mathrm{BL}}}}{2 \sin \theta_{i_{m}^{\mathrm{BL}}}}+\frac{b_{i_{m}^{\mathrm{BR}}}}{2 \sin \theta_{i_{m} \mathrm{BR}}}}{\tan \theta_{m}}-\frac{h_{i_{m}^{\mathrm{CR}}}}{2}\right| \leqslant 1
$$


The stress constraint is split into two constraints to account for the absolute value and the variables belonging to different design entities are separated:

$$
\begin{aligned}
& \underbrace{\frac{\Delta N_{m}}{2 \tan \theta_{m}} g_{m}}_{\text {gap size }}+\underbrace{\frac{\Delta N_{m} b_{i_{m}^{\mathrm{BL}}}}{4 \tan \theta \sin \theta_{i_{m}^{\mathrm{BL}}}}}_{\text {left brace }}+\underbrace{\frac{\Delta N_{m} b_{i_{m}^{\mathrm{BR}}}}{4 \tan \theta \sin \theta_{i_{m}^{\mathrm{BR}}}}}_{\text {right brace }}+\underbrace{\left(\frac{-\Delta N_{m} h_{i_{m}^{\mathrm{CR}}}}{4}-f_{\mathrm{yc}} W_{\mathrm{pl}, y, i_{m}^{\mathrm{CR}}}+\frac{\left|N_{i_{m}^{\mathrm{CR}}}\right| W_{\mathrm{pl}, y, i_{m}^{\mathrm{CR}}}}{A_{i_{m}^{\mathrm{CR}}}}\right)}_{\text {right chord }} \leqslant 0 \\
& \underbrace{-\frac{\Delta N_{m}}{2 \tan \theta_{m}} g_{m}}_{\text {gap size }}-\underbrace{\frac{\Delta N_{m} b_{i_{m}^{\mathrm{BL}}}}{4 \tan \theta \sin \theta_{i_{m}^{\mathrm{BL}}}}}_{\text {left brace }}-\underbrace{\frac{\Delta N_{m} b_{i_{m}^{\mathrm{BR}}}}{4 \tan \theta \sin \theta_{i_{m}^{\mathrm{BR}}}}}_{\text {right brace }}+\underbrace{\left(\frac{\Delta N_{m} h_{i_{m}^{\mathrm{CR}}}}{4}-f_{\mathrm{yc}} W_{\mathrm{pl}, y, i_{m}^{\mathrm{CR}}}+\frac{\left|N_{i_{m}^{\mathrm{CR}}}\right| W_{\mathrm{pl}, y, i_{m}^{\mathrm{CR}}}}{A_{i_{m}^{\mathrm{CR}}}}\right)}_{\text {right chord }} \leqslant 0
\end{aligned}
$$

In reality, multiple profiles are available for each member. For the chord at the right hand side of joint $m$, equations (62) and (63) are then rewritten as:

$$
\begin{aligned}
& \frac{\Delta N_{m}}{2 \tan \theta} g_{m}+\sum_{j \in \mathcal{C}_{i_{m}^{\mathrm{BL}}}} \frac{\Delta N_{m} b_{i_{m}^{\mathrm{BL}} j}}{4 \tan \theta \sin \theta_{i_{m}^{\mathrm{BL}}}} y_{i_{m}^{\mathrm{BL}} j}+\sum_{j \in \mathcal{C}_{i i_{m}^{\mathrm{BR}}}} \frac{\Delta N_{m} b_{i_{m}^{\mathrm{BR}} j}}{4 \tan \theta \sin \theta_{i_{m}^{\mathrm{BR}}}} y_{m} i_{m}^{\mathrm{BR}} j \\
& +\sum_{j \in \mathcal{C}_{i_{m}^{\mathrm{CR}}}}\left(\frac{-\Delta N_{m} h_{i_{m}^{\mathrm{CR} j} j}}{4}-f_{\mathrm{yc}} W_{\mathrm{pl}, y, i_{m}^{\mathrm{CR} j}}+\frac{\left|N_{i_{m}^{\mathrm{CR}}}\right| W_{\mathrm{pl}, y, i_{m}^{\mathrm{CR} j} j}}{A_{i_{m}^{\mathrm{CR} j} j}}\right) y_{i_{m}^{\mathrm{CR} j}} \leqslant 0 \quad \forall m \in \mathcal{J}_{\mathrm{CR}} \\
& -\frac{\Delta N_{m}}{2 \tan \theta} g_{m}-\sum_{j \in \mathcal{C}_{i_{m}^{\mathrm{BL}}}} \frac{\Delta N_{m} b_{i_{m}^{\mathrm{BL}} j}}{4 \tan \theta \sin \theta_{i_{m}^{\mathrm{BL}}}} y_{i_{m}^{\mathrm{BL}} j}-\sum_{j \in \mathcal{C}_{i_{m}^{\mathrm{BR}}}} \frac{\Delta N_{m} b_{i_{m}^{\mathrm{BR}} j}}{4 \tan \theta \sin \theta_{i_{m}^{\mathrm{BR}}}} y_{i_{m}^{\mathrm{BR}} j} \\
& +\sum_{j \in \mathcal{C}_{i_{m}^{\mathrm{CR}}}}\left(\frac{\Delta N_{m} h_{i_{m}^{\mathrm{CR}} j}}{4}-f_{\mathrm{yc}} W_{\mathrm{pl}, y, i_{m}^{\mathrm{CR}} j}+\frac{\left|N_{i_{m}^{\mathrm{CR}}}\right| W_{\mathrm{pl}, y, i_{m}^{\mathrm{CR}} j}}{A_{i_{m}^{\mathrm{CR}} j}}\right) y_{i_{m}^{\mathrm{CR}} j} \leqslant 0 \quad \forall m \in \mathcal{J}_{\mathrm{CR}}
\end{aligned}
$$

For the chords at the left side of joint $m$, equations (64) and (65) are reformulated in the same way, now using the index $i_{m}^{\mathrm{CR}}$ instead of $i_{m}^{\mathrm{CL}}$ and $\mathcal{J}_{\mathrm{CR}}$ instead of $\mathcal{J}_{\mathrm{CL}}$.

As mentioned in the previous section and expressed by equations (8) and (9), the lateral torsional buckling resistance for members bent in the plane of highest flexural rigidity should be checked along the $\mathrm{x}$-axis and along the z-axis. In this case only the top chords must be checked. The constraints for the buckling resistance of the top chord members are reformulated as follows: 


$$
\begin{aligned}
& \frac{\Delta N_{m}}{2 \tan \theta} g_{m}+\sum_{j \in \mathcal{C}_{i_{m}^{\mathrm{BL}}}} \frac{\Delta N_{m} b_{i_{m}^{\mathrm{BL}} j}}{4 \tan \theta \sin \theta_{i_{m}^{\mathrm{BL}}}} y_{i_{m}^{\mathrm{BL}} j}+\sum_{j \in \mathcal{C}_{i_{m}^{\mathrm{BR}}}} \frac{\Delta N_{m} b_{i_{m}^{\mathrm{BR}} j}}{4 \tan \theta \sin \theta_{i_{m}^{\mathrm{BR}}}} y_{i_{m}^{\mathrm{BR}} j} \\
& +\sum_{j \in \mathcal{C}_{i_{m}^{\mathrm{CR}}}}\left(\frac{-\Delta N_{m} h_{i_{m}^{\mathrm{CR}}}}{4}-\frac{\left(1-\frac{N_{i_{m}^{\mathrm{CR}}}}{\chi_{y, i} i_{m}^{\mathrm{CR}} f_{\mathrm{yc}} A_{i_{m} \mathrm{CR}_{j}}}\right) f_{\mathrm{yc}} W_{\mathrm{pl}, \mathrm{y}, i_{m}^{\mathrm{CR} j}}}{\frac{k_{y y, i m_{m}}}{\chi_{\mathrm{LT}, i_{m}^{\mathrm{CR} j}}}}\right) y_{i_{m}^{\mathrm{CR} j}} \leqslant 0 \quad \forall m \in \mathcal{J}_{\mathrm{TJ}} \cap \mathcal{J}_{\mathrm{CR}} \\
& -\frac{\Delta N_{m}}{2 \tan \theta} g_{m}-\sum_{j \in \mathcal{C}_{i_{m}^{\mathrm{BL}}}} \frac{\Delta N_{m} b_{i_{m}^{\mathrm{BL}} j}}{4 \tan \theta \sin \theta_{i_{m}^{\mathrm{BL}}}} y_{i_{m}^{\mathrm{BL}} j}-\sum_{j \in \mathcal{C}_{i_{m}^{\mathrm{BR}}}} \frac{\Delta N_{m} b_{i_{m}^{\mathrm{BR}} j}}{4 \tan \theta \sin \theta_{i_{m}^{\mathrm{BR}}}} y_{i_{m}^{\mathrm{BR}} j} \\
& +\sum_{j \in \mathcal{C}_{i \mathrm{CR}}}\left(\frac{\Delta N_{m} h_{i_{m}^{\mathrm{CR} j} j}}{4}-\frac{\left(1-\frac{N_{i_{m}^{\mathrm{CR}}}}{\chi_{y, i_{m}^{\mathrm{CR} j} j} f_{\mathrm{yc}} A_{i_{m}^{\mathrm{CR} j} j}}\right) f_{\mathrm{yc}} W_{\mathrm{pl}, \mathrm{y}, i_{m}^{\mathrm{CR}} j}}{\frac{k_{y y, i, i \mathrm{CR}}}{\chi_{\mathrm{LT}, i i_{m}^{\mathrm{CR} j}}}}\right) y_{i_{m}^{\mathrm{CR} j} j} \leqslant 0 \quad \forall m \in \mathcal{J}_{\mathrm{TJ}} \cap \mathcal{J}_{\mathrm{CR}}
\end{aligned}
$$

where $\chi_{y, i_{m}^{\mathrm{CR}} j}$ and $\chi_{\mathrm{LT}, i_{m}^{\mathrm{CR}} j}$ are the reduction factors for the resistance of the chord at the right-hand side of the joint due to in-plane flexural buckling and lateral torsional buckling, respectively, and $k_{y y, i_{m}^{\mathrm{CR}} j}$ is an interaction factor. The buckling constraints for the chords at the left side of the joint are reformulated in the same way, now using the index $i_{m}^{\mathrm{CR}}$ instead of $i_{m}^{\mathrm{CL}}$ and $\mathcal{J}_{\mathrm{CR}}$ instead of $\mathcal{J}_{\mathrm{CL}}$. The buckling constraints along the z-axis are reformulated in the same way, by replacing the reduction factor due to in-plane flexural buckling $\chi_{y, i_{m}^{\mathrm{CR}} j}$ with the reduction factor due to outof-plane buckling $\chi_{z, i i_{m}^{\mathrm{CR}} j}$ and the interaction factor $k_{y y, i_{m}^{\mathrm{CR}} j}$ with the interaction factor $k_{z y, i i_{m}^{\mathrm{CR}} j}$. All interaction factors are calculated according to the method described in annex B of Eurocode 3.

For the braces, the stress constraints as given by equation (2), and the in-plane and out-of-plane flexural buckling, torsional buckling and torsional-flexural buckling constraints as given by equations (4) to (7) remain the same as in the previous section:

$$
\begin{aligned}
& N_{i} y_{i j} \leqslant f_{\mathrm{yb}} A_{i j} y_{i j} \quad \forall i \in \mathcal{M}_{\mathrm{B}}, \forall j \in \mathcal{C}_{i} \\
& N_{i} y_{i j} \geqslant-\min \left(1, \chi_{y}, \chi_{z}\right) f_{\mathrm{yb}} A_{i j} y_{i j} \quad \forall i \in \mathcal{M}_{\mathrm{B}}, \forall j \in \mathcal{C}_{i}
\end{aligned}
$$

\section{Joint constraints}

The joint geometry constraints given by equations (10) to (18) and (20) to (28) and those related to the cross-section classes are imposed by only including allowable sections in the profile catalog.

The geometry constraints for the top chords given by equation (19) are reformulated as:

$$
\sum_{j \in \mathcal{C}_{i m}^{\mathrm{BL}}} t_{i_{m}^{\mathrm{BL}} j} y_{i_{m}^{\mathrm{BL}} j}+\sum_{j \in \mathcal{C}_{i m}^{\mathrm{BR}}} t_{i_{m}^{\mathrm{BR}} j} y_{i_{m}^{\mathrm{BR}} j}-g_{m} \leqslant 0 \quad \forall m \in \mathcal{J}_{\mathrm{TJ}}
$$

where $i_{m}^{\mathrm{BL}}$ is the index of the left brace in joint $m$, and $i_{m}^{\mathrm{BR}}$ is the index of the right brace in joint $m$.

The geometry constraints for the bottom chords given by equations (29) and (31) are reformulated as:

$$
\begin{array}{cc}
\sum_{j \in \mathcal{C}_{i_{\mathrm{BC}}}} 0.25 b_{i_{\mathrm{BC}} j} y_{i_{\mathrm{BC}} j}-\sum_{j \in \mathcal{C}_{i_{\mathrm{B}}}} b_{i_{\mathrm{B}} j} y_{i_{\mathrm{B}} j} \leqslant 0 & \forall i_{\mathrm{BC}} \in \mathcal{M}_{\mathrm{B} C}, i_{\mathrm{B}} \in \mathcal{M}_{\mathrm{B}} \\
g_{m}+\sum_{j \in \mathcal{C}_{i_{m}^{\mathrm{BR}}}} b_{i_{m}^{\mathrm{BR}} j} y_{i_{m}^{\mathrm{BR}} j}=0 & \forall m \in \mathcal{J}_{\mathrm{BJ}}
\end{array}
$$


where $i_{m}^{\mathrm{BR}}$ is the index of the overlapping brace in joint $m$.

The geometry constraints for the braces given by equation (30) are reformulated as:

$$
\sum_{j \in \mathcal{C}_{i m}^{\mathrm{BL}}} 0.75 b_{i_{m}^{\mathrm{BL} j}} y_{i_{m}^{\mathrm{BL}} j}-\sum_{j \in \mathcal{C}_{i m}^{\mathrm{BR}}} b_{i_{m}^{\mathrm{BR}} j} y_{i_{m}^{\mathrm{BR}} j} \leqslant 0 \quad \forall m \in \mathcal{J}_{\mathrm{BJ}}
$$

The design resistance for chord web failure of the top joints given by equation (32) contains the effective width for the web of the chord $b_{\mathrm{w}}$, which has to be calculated as the minimum of two expressions. The chord web failure constraints for the top joints are therefore reformulated into two linear constraints. For the braces at the right hand side of the top joints, the first constraint given by equation (32) is reformulated as:

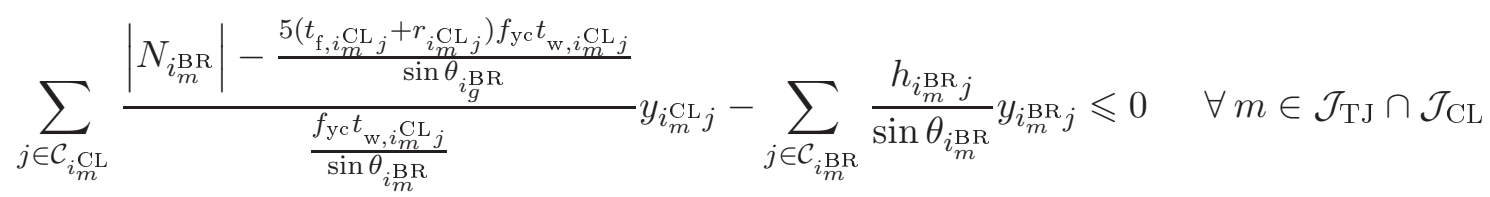

The second constraint given by equation (32) is reformulated as:

$$
\sum_{j \in \mathcal{C}_{i m}^{\mathrm{CL}}} \frac{\left|N_{i_{m}^{\mathrm{BR}}}\right|-\frac{10\left(t_{\mathrm{f}, i_{m}^{\mathrm{CL}} j}+r_{i_{m}^{\mathrm{CL}} j}\right) f_{\mathrm{yc}} t_{\mathrm{w}, i} i_{m}^{\mathrm{CL}} j}{\sin \theta_{i_{m}^{\mathrm{BR}}}}}{\frac{f_{\mathrm{yc}} t_{\mathrm{w}, i} i_{m}^{\mathrm{CL}} j}{\sin \theta_{i_{m}^{\mathrm{BR}}}}} y_{i_{m}^{\mathrm{CL}} j}-\sum_{j \in \mathcal{C}_{i_{m}^{\mathrm{BR}}}} 2 t_{i_{m}^{\mathrm{BR}} j} y_{i_{m}^{\mathrm{BR}} j} \leqslant 0 \quad \forall m \in \mathcal{J}_{\mathrm{TJ}} \cap \mathcal{J}_{\mathrm{CL}}
$$

where $i_{m}^{\mathrm{BR}}$ is the index of the right brace in joint $m$, and $i_{m}^{\mathrm{CL}}$ is the index of the left chord in joint $m$. We could as well have used the right chord, as the same section is used for all chord members. For the top left joint, $i_{m}^{\mathrm{CL}}$ is replaced by $i_{m}^{\mathrm{CR}}$ since there is no left chord. For the braces at the left hand side of the top joints, the chord web failure constraints are reformulated in the same way by replacing $i_{m}^{\mathrm{BR}}$ by $i_{m}^{\mathrm{BL}}$ in equations (74) and (75).

The brace failure constraints for the braces at the right-hand side of the top joints given by equation (34) are reformulated into two linear constraints:

$$
\begin{aligned}
\sum_{j \in \mathcal{C}_{i_{m}^{\mathrm{BR}}}} \frac{\left|N_{i_{m}^{\mathrm{BR}}}\right|}{2 f_{\mathrm{yb}} t_{i_{m}^{\mathrm{BR} j}}} y_{i_{m}^{\mathrm{BR} j}}-\sum_{j \in \mathcal{C}_{i \mathrm{CL}}}\left(t_{\mathrm{w}, i_{m}^{\mathrm{CL} j} j}+2 r_{i_{m}^{\mathrm{CL}} j}+7 t_{\mathrm{f}, i_{m}^{\mathrm{CL}} j} \frac{f_{\mathrm{yc}}}{f_{\mathrm{yb}}}\right) y_{i_{m}^{\mathrm{CL}} j} \leqslant 0 & \forall m \in \mathcal{J}_{\mathrm{TJ}} \\
\left|N_{i_{m}^{\mathrm{BR}}}\right|-\sum_{j \in \mathcal{C}_{i} \mathrm{BR}} 2 f_{\mathrm{yb}} t_{i_{m}^{\mathrm{BR}} j}\left(b_{i_{m}^{\mathrm{BR} j}}+h_{i_{m}^{\mathrm{BR}} j}-2 t_{i_{m}^{\mathrm{BR}} j}\right) y_{i_{m}^{\mathrm{BR} j}} \leqslant 0 & \forall m \in \mathcal{J}_{\mathrm{TJ}}
\end{aligned}
$$

where $f_{\mathrm{yb}}$ is the yield strength of the braces. For the braces at the left hand side of the top joints, the brace failure constraints are reformulated in the same way by replacing $i_{m}^{\mathrm{BR}}$ by $i_{m}^{\mathrm{BL}}$ in equations (76) and (77).

The resistance to chord shear failure for the top joints given by equations (36) and (37) does not have to be checked for the center top joint. The chord shear failure constraints for the other top joints are reformulated as: 


$$
\begin{aligned}
& \left|N_{i_{m}^{\mathrm{BR}}}\right| \leqslant \sum_{j \in \mathcal{C}_{i_{m}^{\mathrm{CL}}}}\left(\frac{f_{\mathrm{yc}} A_{\mathrm{v}, i_{m}^{\mathrm{CL}} j}}{\sqrt{3} \sin \theta_{i_{m}^{\mathrm{BR}}}} \frac{1}{\gamma_{\mathrm{M} 5}}\right) y_{i_{m}^{\mathrm{CL} j} j} \quad \forall m \in \mathcal{J}_{\mathrm{TJ}} \\
& \left|N_{i_{m}^{\mathrm{BL}}}\right| \leqslant \sum_{j \in \mathcal{C}_{i_{m}^{\mathrm{CL}}}}\left(\frac{f_{\mathrm{yc}} A_{\mathrm{v}, i i_{m}^{\mathrm{CL} j}}}{\sqrt{3} \sin \theta_{i_{m}^{\mathrm{BL}}}} \frac{1}{\gamma_{\mathrm{M} 5}}\right) y_{i_{m}^{\mathrm{CL} j} j} \quad \forall m \in \mathcal{J}_{\mathrm{TJ}} \\
& \left|N_{i_{m}^{\mathrm{CL}}}\right| \leqslant \sum_{j \in \mathcal{C}_{i_{m}^{\mathrm{CL}}}}\left(\left[\left(A_{i_{m}^{\mathrm{CL}} j}-A_{\mathrm{v}, i_{m}^{\mathrm{CL}} j}\right) f_{\mathrm{yc}}+A_{\mathrm{v}, i_{m}^{\mathrm{CL}} j} f_{\mathrm{yc}} \sqrt{1-\left(\frac{\gamma_{\mathrm{M} 5} \sqrt{3} \max \left(N_{i_{m}^{\mathrm{BL}}} \sin \theta_{i_{m}^{\mathrm{BL}}}, N_{i_{m}^{\mathrm{BR}}} \sin \theta_{i_{m}^{\mathrm{BR}}}\right)}{f_{\mathrm{yc}} A_{\mathrm{v}, i_{\mathrm{g}} \mathrm{CL}_{j}}}\right)^{2}} \frac{1}{\gamma_{\mathrm{M} 5}}\right) y_{i_{m}^{\mathrm{CL} j}}\right. \\
& \forall m \in \mathcal{J}_{\mathrm{TJ}} \cap \mathcal{J}_{\mathrm{CL}}
\end{aligned}
$$

For the bottom joints, only brace failure of the overlapping brace has to be checked as given by equation (38). The design resistance for brace failure of the bottom joints contains the effective width $b_{\mathrm{e}, \mathrm{ov}}$ of the connection between the overlapping brace and the overlapped brace, which has to be calculated as the minimum of two expressions. The brace failure constraints for the bottom joints are therefore reformulated into two linear constraints. The first constraint is reformulated as:

$$
\sum_{j \in \mathcal{C}_{i m}^{\mathrm{BR}}} \frac{\left|N_{i_{m}^{\mathrm{BR}}}\right|-f_{\mathrm{yb}} t_{i_{m}^{\mathrm{BR}} j}\left(b_{i_{m}^{\mathrm{BR}} j}+2 h_{i_{m}^{\mathrm{BR}} j}-4 t_{i_{m}^{\mathrm{BR}} j}\right)}{b_{i_{m}^{\mathrm{BR}} j}} y_{i_{m}^{\mathrm{BR}} j}-\sum_{j \in \mathcal{C}_{i_{m}^{\mathrm{BL}}}} \frac{10 f_{\mathrm{yb}} t_{i_{m}^{\mathrm{BL}} j}}{\frac{b_{i_{m}^{\mathrm{BL}} j}}{t_{i_{m}^{\mathrm{BL}} j}}} y_{i_{m}^{\mathrm{BL}} j} \leqslant 0 \quad \forall m \in \mathcal{J}_{\mathrm{BJ}}
$$

The second constraint is reformulated as:

$$
\left|N_{i_{m}^{\mathrm{BR}}}\right|-\sum_{j \in \mathcal{C}_{i \mathrm{BR}}} f_{\mathrm{yb}^{\mathrm{BR}}} t_{i_{m}^{\mathrm{BR}} j}\left(2 b_{i_{m}^{\mathrm{BR}} j}+2 h_{i_{m}^{\mathrm{BR}} j}-4 t_{i_{m}^{\mathrm{BR}} j}\right) y_{i_{m}^{\mathrm{BR}} j} \leqslant 0 \quad \forall m \in \mathcal{J}_{\mathrm{BJ}}
$$

\section{Results}

The MILP of the N-truss given by equations (50) to (56), and (64) to (82) considering displacement, member and joint constraints consist of 1143 design variables, including 1112 binary variables, 21 continuous nodal displacement variables and 10 continuous gap variables. The MILP consists of 4751 constraints, including 232 additional member and joint constraints, and 36344 non-zeros. The MILP is again solved by means of Gurobi 5.6 using the same computer as for the previous case. The optimal solution is again found in the preprocessing stage in less than 0.2 seconds. When the MILP is solved without performing a presolve and without generating cuts, the problem can no longer be solved at the root node. In this case, 1110 nodes are explored, and the computation time is 1.3 seconds. The results are given in table 8 . The total weight of the N-truss is $2091.0 \mathrm{~kg}$.

When the optimization problem is solved taking into account the displacement constraints, member constraints, and joint constraints, the design variables also include the gaps in order to be able to implement the joint geometry constraints and to take into account the eccentricity moments. The optimal gap values are given in table 9. The displacements are given in table 10. Also in this case the displacement constraints are not critical. The utilization ratio for each constraint is given in table 11. In this case all the utilization ratios - including those related to the joint constraints are smaller than 1.

The weight of the optimized structure is about $15 \%$ higher than in the case where joint constraints are not considered. This shows that taking into account joint constraints during the optimization has a significant impact on the optimized design. 


\section{CONCLUSION}

For a practical truss design optimization problem, three types of constraints have to be taken into account: displacement constraints, member constraints, and joint constraints. Most optimization methods do not take into account the joint constraints. As a consequence, the joint constraints must be checked after optimization and the joints may need to be strengthened. This paper verifies the importance of joint constraints in the optimal design of trusses and presents a method to account for joint constraints during the optimization. The optimization problem is solved by reformulating it as a mixed-integer linear program (MILP). The vector of design variables consists of (1) binary decision variables which select a section from a catalog for each member, (2) nodal displacement variables, and (3) joint gaps when joint constraints are taken into account. The MILP is solved with the cut-and-branch method which is implemented in the Gurobi optimizer. The advantage of this approach is that the problem can be solved to global optimality.

This paper makes a comparison between the results obtained after optimization of a statically determinate steel N-truss girder with and without joint constraints. The results show that taking into account joint constraints during the optimization leads to a result with a weight that is $15 \%$ higher than in the case where these constraints are not considered. When only displacement and member constraints are taken into account during the optimization, one should be very careful when checking the joint constraints and adapting the optimized design a posteriori in order to satisfy all constraints, since the joint constraints have a large impact on the total weight of the structure. Taking into account joint constraints during the optimization therefore leads to a cost reduction at two levels: in terms of engineering cost (no manual postprocessing step is needed) as well as fabrication cost (joint strengthening is avoided).

\section{References}

ArcelorMittal (2015). ArcelorMittal Sections and Merchant Bars, $<$ http://amsections.arcelormittal.com//products-services/products-ranges.html $>$. Accessed Apr., 2015.

Arora, J. and Wang, Q. (2005). "Review of formulations for structural and mechanical system optimization." Structural and Multidisciplinary Optimization, 30(4), 251-272.

Balling, R. (1991). "Optimal steel frame design by simulated annealing." Journal of structural engineering, 117(6), 1780-1795.

Camp, C., Bichon, B., and Stovall, S. (2005). "Design of steel frames using ant colony optimization." Journal of structural engineering, 131(7), 369-379.

Camp, C., Pezeshk, S., and Guozhong, C. (1998). "Optimized design of two-dimensional structures using a genetic algorithm." Journal of structural engineering, 124(5), 551-559.

European Committee for Standardization (2005a). Eurocode 3: Design of steel structures - Part 1-1: General rules and rules for buildings.

European Committee for Standardization (2005b). Eurocode 3: Design of steel structures - Part 1-8: Design of joints.

Gandomi, A., Yang, X., and Alavi, A. (2011). "Mixed variable structural optimization using firefly algorithm." Computers \& Structures, 89(23), 2325-2336. 
Grossmann, I., Voudouris, V., and Ghattas, O. (1992). "Mixed-integer linear programming reformulations for some nonlinear discrete design optimization problems." Recent advances in global optimization, C. Floudas and P. Pardalos, eds., Princeton University Press, 478-512.

Gurobi Optimization Inc. (2013). Gurobi Optimizer Reference Manual, <http://www.gurobi.com/documentation/5.6/reference-manual>. Accessed Aug., 2014.

Haftka, R. (1985). "Simultaneous analysis and design." AIAA Journal, 23(7), 1099-1103.

Mela, K. (2013). "Mixed variable formulations for truss topology optimization." Ph.D. thesis, Tampere university of technology, Tampere, Finland.

Mela, K. and Koski, J. (2013). "Distributed loads in truss topology optimization." Proceedings of the 10th World Congress on Structural and Multidisciplinary Optimization, Orlando, USA (May).

Mueller, K., Liu, M., and Burns, S. (2002). "Fully stressed design of frame structures and multiple load paths." Journal of structural engineering, 128(6), 806-814.

Patnaik, S., Gendy, A., Berke, L., and Hopkins, D. (1998). "Modified fully utilized design (MFUD) method for stress and displacement constraints." International Journal for Numerical Methods in Engineering, 41(7), 1171-1194.

Patnaik, S. and Hopkins, D. (1998). "Optimality of a fully stressed design." Computer methods in applied mechanics and engineering, 165, 215-221.

Rajeev, S. and Krishnamoorthy, C. (1992). "Discrete optimization of structures using genetic algorithms." Journal of Structural Engineering, 118(5), 1233-1250.

Rasmussen, M. and Stolpe, M. (2008). "Global optimization of discrete truss topology design problems using a parallel cut-and-branch method." Computers \& Structures, 86(13), 1527-1538.

Razani, R. (1965). "Behavior of fully-stressed design of structures and its relationship to minimumweight design." AIAA Journal, 3(12), 2262-2268.

Sonmez, M. (2011). "Discrete optimum design of truss structures using artificial bee colony algorithm." Structural and Multidisciplinary Optimization, 43(1), 85-97.

Stolpe, M. (2007). "On the refomulation of topology optimization problems as linear or convex quadratic mixed 0-1 programs." Optimization in Engineering, (8), 163-192.

Stolpe, M. and Svanberg, K. (2003). "Modelling topology optimization problems as linear mixed 0-1 programs." International Journal for Numerical Methods in Engineering, 57, 723-739.

Thanedar, P. and Vanderplaats, G. (1995). "Survey of discrete variable optimization for structural design." Journal of structural engineering, 121(2), 301-306.

van Eldik, C. (2006). Wegwijzer constructiestaal. Zoetermeer Bouwen met staal.

Venter, G. and Sobieszczanski-Sobieski, J. (2003). "Particle swarm optimization." AIAA Journal, 41(8), 1583-1589.

Wardenier, J., Kurobane, Y., Packer, van der Vegte, G., and Zhao, X.-L. (2008). Design guide for circular hollow section (CHS) joints under predominantly static loading. CIDECT and LSS Verlag. 
Wardenier, J., Kurobane, Y., Packer, J., Dutta, D., and Yeomans, N. (1992). Design guide for circular hollow section (CHS) joints under predominantly static loading. CIDECT and Verlag TÜV Rheinland GmbH.

\section{Tables}

Table 1: HEA profile catalog

\begin{tabular}{cc|cc|cc}
\hline Index & Name & Index & Name & Index & Name \\
\hline 1 & HEA 100 & 9 & HEA 260 & 17 & HEA 500 \\
2 & HEA 120 & 10 & HEA 280 & 18 & HEA 550 \\
3 & HEA 140 & 11 & HEA 300 & 19 & HEA 600 \\
4 & HEA 160 & 12 & HEA 320 & 20 & HEA 650 \\
5 & HEA 180 & 13 & HEA 340 & 21 & HEA 700 \\
6 & HEA 200 & 14 & HEA 360 & 22 & HEA 800 \\
7 & HEA 220 & 15 & HEA 400 & 23 & HEA 900 \\
8 & HEA 240 & 16 & HEA 450 & 24 & HEA 1000 \\
\hline
\end{tabular}

Table 2: UPN profile catalog

\begin{tabular}{cc|cc|cc}
\hline Index & Name & Index & Name & Index & Name \\
\hline 1 & UPN 50 & 7 & UPN 160 & 13 & UPN 280 \\
2 & UPN 65 & 8 & UPN 180 & 14 & UPN 300 \\
3 & UPN 80 & 9 & UPN 200 & 15 & UPN 320 \\
4 & UPN 100 & 10 & UPN 220 & 16 & UPN 350 \\
5 & UPN 120 & 11 & UPN 240 & 17 & UPN 380 \\
6 & UPN 140 & 12 & UPN 260 & 18 & UPN 400 \\
\hline
\end{tabular}


Table 3: square RHS profile catalog

\begin{tabular}{|c|c|c|c|c|c|}
\hline Index & Name & Index & Name & Index & Name \\
\hline 1 & RHS 20x20x2 & 29 & RHS 80x80x3 & 57 & RHS 140x140x8 \\
\hline 2 & RHS $25 \times 25 \times 2$ & 30 & RHS $80 \times 80 x 4$ & 58 & RHS 140x140x10 \\
\hline 3 & RHS $25 \times 25 \times 3$ & 31 & RHS 80x80x5 & 59 & RHS 150x150x4 \\
\hline 4 & RHS 30x30x2 & 32 & RHS 80x80x6 & 60 & RHS 150x150x5 \\
\hline 5 & RHS 30x30x3 & 33 & RHS $80 \times 80 \times 8$ & 61 & RHS 150x150x6 \\
\hline 6 & RHS 30x30x4 & 34 & RHS 90x90x3 & 62 & RHS 150x150x8 \\
\hline 7 & RHS $35 \times 35 \times 2$ & 35 & RHS 90x90x4 & 63 & RHS 150x150x10 \\
\hline 8 & RHS $35 \times 35 \times 3$ & 36 & RHS 90x90x5 & 64 & RHS 160x160x5 \\
\hline 9 & RHS $40 \times 40 \times 2$ & 37 & RHS 90x90x6 & 65 & RHS 160x160x6 \\
\hline 10 & RHS 40x40x3 & 38 & RHS 100x100x3 & 66 & RHS 160x160x8 \\
\hline 11 & RHS $40 \times 40 \times 4$ & 39 & RHS 100x100x4 & 67 & RHS 160x160x10 \\
\hline 12 & RHS $45 \times 45 \times 2$ & 40 & RHS 100x100x5 & 68 & RHS 180x180x6 \\
\hline 13 & RHS $45 \times 45 \times 3$ & 41 & RHS 100x100x6 & 69 & RHS 180x180x8 \\
\hline 14 & RHS $45 \times 45 \times 4$ & 42 & RHS 100x100x8 & 70 & RHS 180x180x10 \\
\hline 15 & RHS 50x50x2 & 43 & RHS 100x100x10 & 71 & RHS $180 \times 180 \times 12.5$ \\
\hline 16 & RHS 50x50x3 & 44 & RHS 110x110x4 & 72 & RHS 200x200x5 \\
\hline 17 & RHS 50x50x4 & 45 & RHS 110x110x5 & 73 & RHS $200 \times 200 \times 6$ \\
\hline 18 & RHS $50 \times 50 \times 5$ & 46 & RHS 120x120x3 & 74 & RHS $200 \times 200 \times 8$ \\
\hline 19 & RHS 60x60x2 & 47 & RHS 120x120x4 & 75 & RHS 200x200x10 \\
\hline 20 & RHS 60x60x3 & 48 & RHS 120x120x5 & 76 & RHS $200 \times 200 \times 12.5$ \\
\hline 21 & RHS $60 \times 60 \times 4$ & 49 & RHS $120 \times 120 \times 6$ & 77 & RHS 220x220x6 \\
\hline 22 & RHS 60x60x5 & 50 & RHS 120x120x8 & 78 & RHS 220x220x8 \\
\hline 23 & RHS 60x60x6 & 51 & RHS 120x120x10 & 79 & RHS 220x220x10 \\
\hline 24 & RHS 70x70x2 & 52 & RHS 125x125x5 & 80 & RHS 250x250x6 \\
\hline 25 & RHS 70x70x3 & 53 & RHS $125 \times 125 \times 6$ & 81 & RHS 250x250x8 \\
\hline 26 & RHS $70 \times 70 \times 4$ & 54 & RHS $140 \times 140 \times 4$ & 82 & RHS 250x250x10 \\
\hline 27 & RHS 70x70x5 & 55 & RHS 140x140x5 & & \\
\hline 28 & RHS 70x70x6 & 56 & RHS 140x140x6 & & \\
\hline
\end{tabular}


Table 4: Sections of the N-truss optimized considering only displacement and member constraints.

\begin{tabular}{|c|c|c|}
\hline Member & Profile & $\mathrm{A}\left[\mathrm{mm}^{2}\right.$ \\
\hline Top chords & HEA 180 & 4530 \\
\hline Bottom chords & UPN 220 & 3740 \\
\hline Brace 11 & RHS 110x110x5 & 2036 \\
\hline Brace 12 & RHS $125 \times 125 \times 5$ & 2336 \\
\hline Brace 13 & RHS 120x120x4 & 1815 \\
\hline Brace 14 & RHS 120x120x4 & 1815 \\
\hline Brace 15 & RHS 100x100x4 & 1495 \\
\hline Brace 16 & RHS 90x90x4 & 1335 \\
\hline Brace 17 & RHS 100x100x3 & 1141 \\
\hline Brace 18 & RHS 70x70x3 & 781 \\
\hline Brace 19 & RHS $70 \times 70 \times 3$ & 781 \\
\hline Brace 20 & RHS $40 \times 40 \times 2$ & 294 \\
\hline Brace 21 & RHS $70 \times 70 \times 2$ & 534 \\
\hline \multicolumn{3}{|c|}{ Weight: $1826.3 \mathrm{~kg}$} \\
\hline
\end{tabular}

Table 5: Nodal displacements of the N-truss optimized considering only displacement and member constraints.

\begin{tabular}{lcccccccccccccc}
\hline Node number & 1 & 2 & 3 & 4 & 5 & 6 & 7 & 8 & 9 & 10 & 11 \\
\hline Horizontal displacement $[\mathrm{mm}]$ & 7.40 & 6.70 & 5.45 & 3.82 & 1.95 & 0 & -6.60 & -6.60 & -5.75 & -4.24 & -2.26 & 0 & \\
Vertical displacement $[\mathrm{mm}]$ & -1.73 & -21.32 & -39.27 & -54.25 & -65.52 & -72.18 & 0 & -19.58 & -37.62 & -52.71 & -64.16 & -70.86 \\
\hline
\end{tabular}

Table 6: Gaps and eccentricities introduced to ensure weldability of the joints of the N-truss optimized considering only displacement and member constraints.

\begin{tabular}{ccc}
\hline Joint number & Gaps $[\mathrm{mm}]$ & Eccentricity $[\mathrm{mm}]$ \\
\hline 1 & 10.0 & 67.9 \\
2 & 8.0 & 67.4 \\
3 & 8.0 & 36.1 \\
4 & 6.0 & 20.0 \\
5 & 5.0 & -17.2 \\
8 & -120.0 & 7.0 \\
9 & -100.0 & 13.5 \\
10 & -100.0 & -7.8 \\
11 & -70.0 & -6.9 \\
12 & -70.0 & -28.1 \\
\hline
\end{tabular}


Table 7: Utilization ratios for the optimized design of the N-truss considering displacement and member constraints.

\begin{tabular}{|c|c|c|c|c|c|c|}
\hline \multirow[b]{2}{*}{ 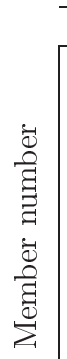 } & \multicolumn{2}{|c|}{ Member constraints } & \multicolumn{4}{|c|}{ Joint constraints } \\
\hline & Member resistance ratio & Stability ratio & 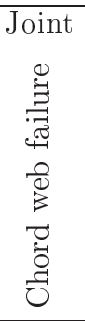 & 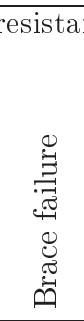 & 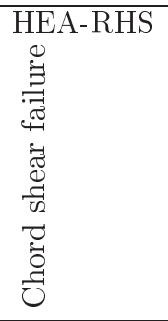 & Joint resistance ratio UPN-RHS \\
\hline 1 & 0.54 & 0.60 & & & 0.39 & \\
\hline 2 & 0.60 & 0.67 & & & 0.53 & \\
\hline 3 & 0.69 & 0.81 & & & 0.67 & \\
\hline 4 & 0.76 & 0.91 & & & 0.75 & \\
\hline 5 & 0.78 & 0.94 & & & & \\
\hline 6 & 0.07 & & & & & \\
\hline 7 & 0.41 & & & & & \\
\hline 8 & 0.71 & & & & & \\
\hline 9 & 0.83 & & & & & \\
\hline 10 & 0.93 & & & & & \\
\hline 11 & 0.89 & 1.00 & 1.01 & 1.49 & 0.96 & \\
\hline 12 & 0.99 & & 0.83 & 1.90 & 0.86 & \\
\hline 13 & 0.90 & 0.99 & 0.87 & 1.68 & 0.82 & 1.01 \\
\hline 14 & 0.99 & & 0.65 & 1.85 & 0.64 & \\
\hline 15 & 0.85 & 0.97 & 0.74 & 1.31 & 0.64 & 1.00 \\
\hline 16 & 0.96 & & 0.47 & 1.32 & 0.46 & \\
\hline 17 & 0.80 & 0.91 & 0.53 & 1.24 & 0.43 & 0.87 \\
\hline 18 & 0.99 & & 0.32 & 1.06 & 0.26 & \\
\hline 19 & 0.70 & 0.91 & 0.37 & 0.75 & 0.25 & 0.80 \\
\hline 20 & 0.87 & & 0.13 & 0.85 & 0.08 & \\
\hline 21 & 0.68 & 0.88 & 0.24 & 0.75 & & 0.77 \\
\hline
\end{tabular}

Table 8: Sections of the N-truss optimized considering displacement, member, and joint constraints.

\begin{tabular}{|c|c|c|}
\hline Member & Profile & $\mathrm{A}\left[\mathrm{mm}^{2}\right]$ \\
\hline Top chords & HEA 200 & 5380 \\
\hline Bottom chords & UPN 220 & 3740 \\
\hline Brace 11 & RHS $100 \times 100 \times 8$ & 2724 \\
\hline Brace 12 & RHS $100 \times 100 \times 10$ & 3257 \\
\hline Brace 13 & RHS 100x100x8 & 2724 \\
\hline Brace 14 & RHS 80x80x8 & 2084 \\
\hline Brace 15 & RHS 90x90x5 & 1636 \\
\hline Brace 16 & RHS $80 \times 80 \times 5$ & 1436 \\
\hline Brace 17 & RHS $80 \times 80 x 4$ & 1175 \\
\hline Brace 18 & RHS 70x70x3 & 781 \\
\hline Brace 19 & RHS $70 \times 70 \times 3$ & 781 \\
\hline Brace 20 & RHS $60 \times 60 \times 3$ & 661 \\
\hline Brace 21 & RHS 60x60x3 & 661 \\
\hline \multicolumn{3}{|c|}{ Weight: $2091.0 \mathrm{~kg}$} \\
\hline
\end{tabular}


Table 9: Gaps and eccentricities introduced to ensure weldability of the joints of the N-truss optimized considering displacement, member, and joint constraints.

\begin{tabular}{ccc}
\hline Joint number & Gap $[\mathrm{mm}]$ & Eccentricity $[\mathrm{mm}]$ \\
\hline 1 & 18.0 & 43.7 \\
2 & 16.0 & 27.6 \\
3 & 10.0 & 16.6 \\
4 & 7.0 & 1.5 \\
5 & 6.0 & -11.6 \\
8 & -100.0 & -0.7 \\
9 & -90.0 & -9.8 \\
10 & -80.0 & -4.8 \\
11 & -70.0 & -6.9 \\
12 & -60.0 & -9.0 \\
\hline
\end{tabular}

Table 10: Nodal displacements of the N-truss optimized considering displacement, member, and joint constraints.

\begin{tabular}{lcccccccccccccc}
\hline Node number & 1 & 2 & 3 & 4 & 5 & 6 & 7 & 8 & 9 & 10 & 11 & 12 \\
\hline Horizontal displacement $[\mathrm{mm}]$ & 6.23 & 5.64 & 4.59 & 3.21 & 1.64 & 0 & -6.60 & -6.60 & -5.75 & -4.24 & -2.26 & 0 & \\
Vertical displacement $[\mathrm{mm}]$ & -1.29 & -18.05 & -34.30 & -48.11 & -58.77 & -62.99 & 0 & -16.88 & -32.79 & -46.61 & -57.42 & -61.92 \\
\hline
\end{tabular}


Table 11: Utilization ratio of the optimized result taking into account displacement, member, and joint constraints.

\begin{tabular}{|c|c|c|c|c|c|c|}
\hline \multirow[b]{2}{*}{ 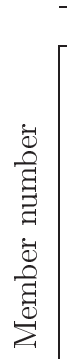 } & \multicolumn{2}{|c|}{ Member constraints } & \multicolumn{4}{|c|}{ Joint constraints } \\
\hline & Member resistance ratio & Stability ratio & 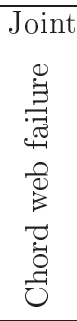 & 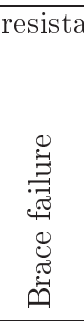 & 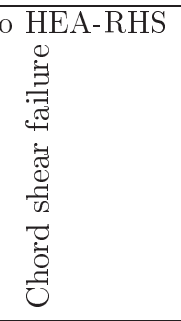 & Joint resistance ratio UPN-RHS \\
\hline 1 & 0.36 & 0.39 & & & 0.30 & \\
\hline 2 & 0.45 & 0.51 & & & 0.44 & \\
\hline 3 & 0.56 & 0.65 & & & 0.56 & \\
\hline 4 & 0.63 & 0.73 & & & 0.63 & \\
\hline 5 & 0.66 & 0.76 & & & & \\
\hline 6 & 0.01 & & & & & \\
\hline 7 & 0.35 & & & & & \\
\hline 8 & 0.68 & & & & & \\
\hline 9 & 0.82 & & & & & \\
\hline 10 & 0.93 & & & & & \\
\hline 11 & 0.67 & 0.78 & 0.90 & 0.86 & 0.91 & \\
\hline 12 & 0.71 & & 0.69 & 0.87 & 0.82 & \\
\hline 13 & 0.60 & 0.70 & 0.81 & 0.77 & 0.80 & 0.56 \\
\hline 14 & 0.86 & & 0.60 & 0.85 & 0.62 & \\
\hline 15 & 0.78 & 0.92 & 0.66 & 0.96 & 0.55 & 0.75 \\
\hline 16 & 0.90 & & 0.43 & 0.97 & 0.39 & \\
\hline 17 & 0.77 & 0.96 & 0.49 & 0.86 & 0.36 & 0.79 \\
\hline 18 & 0.99 & & 0.27 & 0.97 & 0.22 & \\
\hline 19 & 0.70 & 0.91 & 0.31 & 0.68 & 0.21 & 0.80 \\
\hline 20 & 0.39 & & 0.10 & 0.38 & 0.07 & \\
\hline 21 & 0.55 & 0.79 & 0.22 & 0.53 & & 0.61 \\
\hline
\end{tabular}




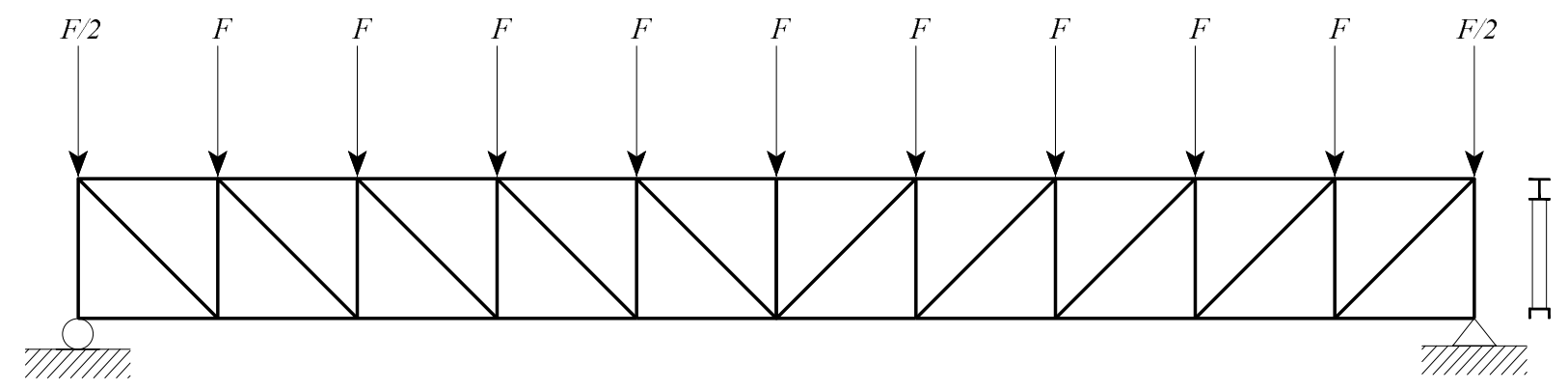

Figure 1: N-truss girder with HEA top chord sections, UPN bottom chord sections, and RHS braces subjected to vertical point loads $F$ and $F / 2$.

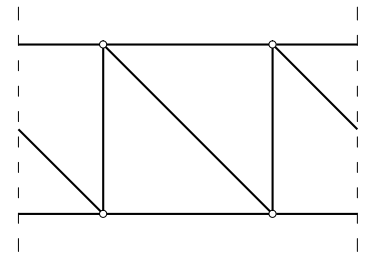

(a) Model 1

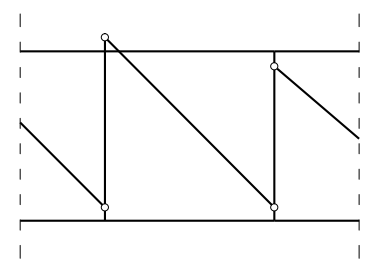

(b) Model 2

Figure 2: These figures show the two different analysis models. Model 1 is a statically determinate truss model where all members are pin connected. Model 2 is a statically indeterminate truss model where the chords are continuous and the braces are pin connected with infinitely stiff members.

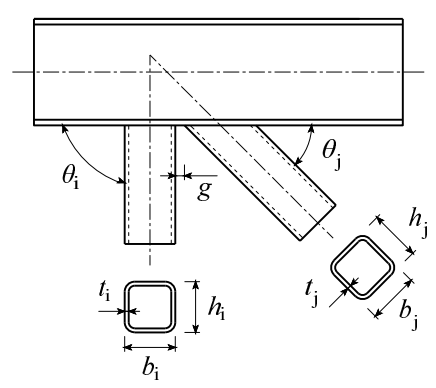

(a)

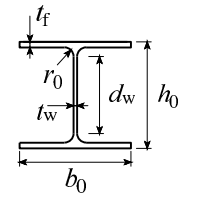

Figure 3: Joints: (a) HEA-RHS joint, (b) UPN-RHS joint.
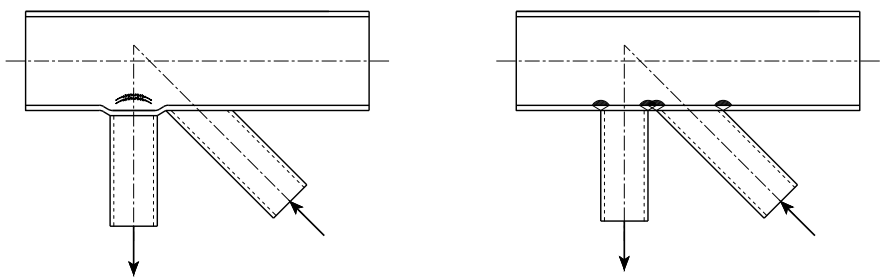

Figure 4: Chord web failure. 


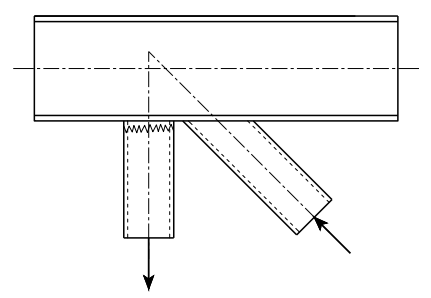

Figure 5: Brace failure.

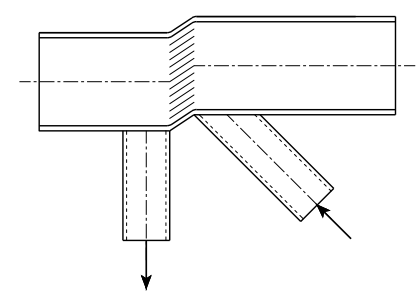

Figure 6: Chord shear failure.

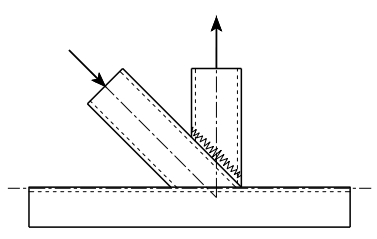

Figure 7: Brace failure.

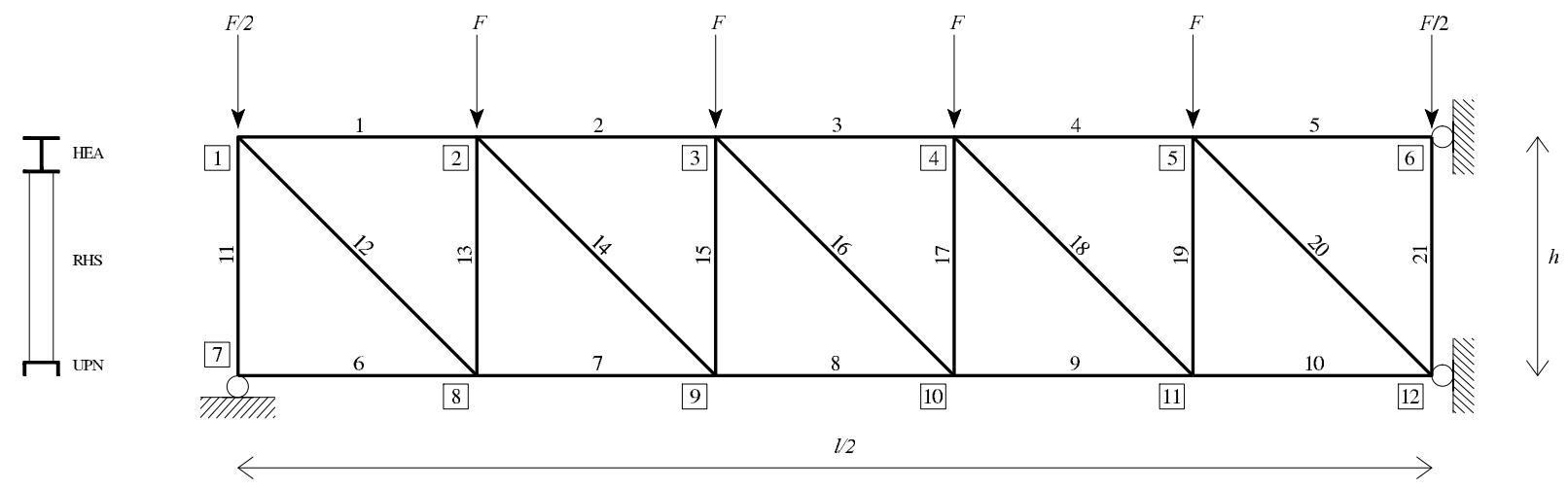

Figure 8: Symmetric half of the N-type truss girder with a span $l$, height $h$ and vertical point loads $F$ and $F / 2$. 